\title{
The Role of Ocular Blood Flow in the Pathogenesis of Glaucomatous Damage
}

\author{
Josef Flammer, MD, ${ }^{1}$ Katarzyna Konieczka, MDํa and Andreas J Flammer, MD² \\ 1. Professor, Department of Ophthalmology, University of Basel; 2. Research Fellow, Division of Cardiovascular Diseases, \\ Department of Internal Medicine, Mayo Clinic and College of Medicine, Rochester
}

\begin{abstract}
Ocular blood flow (OBF) is on average lower in glaucoma patients than in healthy controls. This reduction is more pronounced in normal-tension than in high-tension glaucoma and more distinct in cases with progressing damage as compared to those with stable disease. Besides a secondary component caused by atrophy, there is an important primary component of OBF reduction, which also has a predictive value. The fact that hypoxia-related factors are upregulated in eyes of glaucoma patients indicates oxygen depletion. It is, however, not constant hypoxia, but rather the fluctuation of oxygen supply that leads to tissue damage, likely because of oxidative stress. Low perfusion pressure and disturbed autoregulation are the major causes of insufficient and fluctuating oxygen supply, and both systemic hypotension and disturbed autoregulation are often consequences of the primary vascular dysregulation syndrome. The observed splinter hemorrhages in these patients are a consequence of a local breakdown of the blood-brain or blood-retinal barrier. The often associated vein occlusions can be a consequence of a local vein dysregulation.
\end{abstract}

\section{Keywords}

Glaucoma, ocular blood flow, hypoxia, primary vascular dysregulation, autoregulation, ocular perfusion pressure, splinter hemorrhages, retinal vein occlusion

Disclosure: The authors have no conflicts of interest to declare.

Received: August 8, 2011 Accepted: September 6, 2011 Citation: US Ophthalmic Review, 2011;4(2):84-7 DOI: 10.17925/USOR.2011.04.02.84

Correspondence: Josef Flammer, MD, Professor, Department of Ophthalmology, University of Basel, Mittlere Str 91, CH-4031 Basel, Switzerland. E: Flammer」@uhbs.ch.

For years the question as to whether blood flow plays a role in the pathogenesis of glaucomatous damage has been controversial. The large number of publications on this topic illustrates this and a review is warranted. Herein we discuss the questions asked most often.

\section{Can We Measure Ocular Blood Flow?}

Indeed, it is challenging to measure ocular blood flow (OBF)-several methods are used, but there is no gold standard as yet. Nevertheless, OBF can be measured and different instruments are already in daily use, both for clinical and research purposes. ${ }^{1}$ Although these different techniques quantify blood flow or blood-flow velocity in different vessels using different methods, the outcomes are very often well correlated (see Table 1). ${ }^{2}$

\section{Is Ocular Blood Flow Altered in Glaucoma?}

Even if we acknowledge the limitations of the different technologies used to measure OBF, the results in the literature are unambiguous as the vast majority of studies reveal a reduced OBF in glaucoma patients. In contrast, OBF is normal or even above normal in patients with ocular hypertension. Importantly, OBF is more reduced in normal-tension glaucoma (NTG) patients than in high-tension glaucoma patients. In other words, the lower the intraocular pressure (IOP) at which damage occurs, the higher the probability of finding reduced OBF. Furthermore, $\mathrm{OBF}$ is significantly more reduced in glaucoma patients with progressing disease than in patients with no progression. ${ }^{3}$

\section{Is Ocular Blood Flow Reduction a Cause or a Consequence of Glaucomatous Damage?}

It is obvious that blood flow is reduced in atrophic tissues. Therefore, that there is a secondary component is of little doubt. Interestingly, however, there are also many indications of a primary component. First of all, blood flow reduction can be observed in other parts of the body as well, such as in the nailfold capillaries, ${ }^{4}$ which obviously cannot be a consequence of glaucomatous damage. In addition, reduction of OBF often precedes further glaucomatous damage, which indicates that there must be an important primary component.

\section{Is Reduced Ocular Blood Flow of Predictive Value?}

Several independent studies demonstrated convincingly that a reduced OBF is, indeed, a risk factor for further progression. ${ }^{5}$ In other words, a reduced OBF has an effect similar to that of an increased IOP (Figure 1)

\section{Do We See Signs of Hypoxia in Glaucoma Patients?}

Both in the retina and in the optic nerve head of glaucoma patients, the hypoxia inducible factor $1 \alpha$ (HIF) is increased. ${ }^{6}$ HIF is produced constantly by all types of cells. Under normoxic conditions, HIF is oxidized, and then marked by ubiquitin and degraded by proteasomes. If, however, oxygen concentration is low, degradation is decreased and the concentration of HIF increases. HIF acts as a transcription factor 
and leads to an upregulation of a number of proteins, such as erythropoietin, vascular endothelial growth factor (VEGF), endothelin, and the von Willebrand factor. Correspondingly, all these factors have been described as increased in glaucoma patients.

\section{Is Hypoxia Sufficient to Induce Glaucomatous Optic Neuropathy?}

A slight but constant reduction of oxygen supplied to an organ like the eye interestingly does not lead to major damage. Most tissues have a certain capacity to adapt to hypoxia. In patients with multiple sclerosis (MS) endothelin plasma concentration, for example, is increased markedly and therefore OBF is decreased.? Although a few of these patients get some degree of optic nerve head atrophy, they do not excavate more often than in subjects without MS, a fact that is also true for a number of other diseases, such as polyarthritis. Contrary to the findings in conditions with constant oxygen reduction, unstable, fluctuating OBF is likely to cause glaucomatous damage. ${ }^{8}$ Indeed, variation of OBF correlates with progression of glaucomatous damage.

\section{Why is Ocular Blood Flow Unstable in Glaucoma Patients?}

OBF is unstable if IOP fluctuates on a high enough or blood pressure on a low enough level to exceed temporarily the autoregulation capacity. Indeed, IOP fluctuation is related to both an increase in scotomas and an increase in diffuse visual fields damage. ${ }^{8}$

OBF, however, is also unstable if autoregulation itself is disturbed. Reduced autoregulation occurs particularly in patients who suffer from primary vascular dysregulation (PVD) syndrome. ${ }^{9}$

\section{What is the Role of Blood Pressure?}

Although low blood pressure has been described as a risk factor for glaucomatous damage and its progression for decades, ${ }^{10}$ the majority of ophthalmologists still neglected the role of systemic arterial hypotension. ${ }^{11}$ Not only have recent studies re-emphasized the important contributory role of low blood pressure, but also they have shown that a fluctuation of blood pressure is even more relevant. Finally, the best predictor for glaucomatous damage progression is the fluctuation of perfusion pressure. ${ }^{12}$

\section{Can we Measure Ocular Perfusion Pressure?}

Traditionally, ocular perfusion pressure (OPP) is calculated as the difference between systemic blood pressure (measured in the upper arm) and the retinal venous pressure (assumed to be equal to IOP). Beside the fact that systemic blood pressure is only a relatively weak estimate for retinal arterial pressure, retinal venous pressure is definitively not necessarily equal to IOP. Although this simplification might be acceptable for the majority of healthy people, it is not applicable for glaucomatous patients. ${ }^{13}$ Therefore, to quantify OPP, we should measure blood pressure (arterial and venous pressure) directly in the eye. This can be done with the help of an ophthalmodynamometer.

\section{What are the Signs and Symptoms of a Primary Vascular Dysregulation?}

Unfortunately, there are no pathognomonic symptoms or signs for the diagnosis of PVD. PVD tends to occur more often in females, in people
Table 1: Methods to Measure Ocular Blood Flow

\begin{tabular}{|c|c|c|}
\hline Method & Measuring Field & Outcome \\
\hline \multicolumn{3}{|l|}{ Video-angiography with } \\
\hline Fluorescein & Retinal vessels & $\begin{array}{l}\text { Blood flow velocity } \\
\text { Blood-retinal barrier }\end{array}$ \\
\hline Indocyanine green & Choroidal vessels & Blood flow velocity \\
\hline \multirow[t]{2}{*}{ Color doppler imaging } & Retrobulbar vessels & Blood flow velocity \\
\hline & & Blood flow resistivity \\
\hline \multicolumn{3}{|l|}{ Laser doppler- } \\
\hline Velocimetry & Retinal vessels & Blood flow velocity \\
\hline Flowmetry & ONH or retina & Capillary blood flow \\
\hline Laser speckle flowmetry & $\mathrm{ONH}$ & Capillary blood flow \\
\hline Retinal vessel analyzer & Retinal vessels & Neurovascular coupling \\
\hline Thermography & Cornea & Bulk flow of the eye \\
\hline Ocular blood pressure analyzer & Retina/ONH & $\begin{array}{l}\text { Arterial and venous } \\
\text { ocular blood pressure }\end{array}$ \\
\hline
\end{tabular}

$\mathrm{ONH}=$ optic nerve head.

Figure 1: Higher Intraocular Pressure Results in a Higher Rate of Glaucomatous Damage Progression, Lower Ocular Blood Flow Results in Higher Chance for Progression

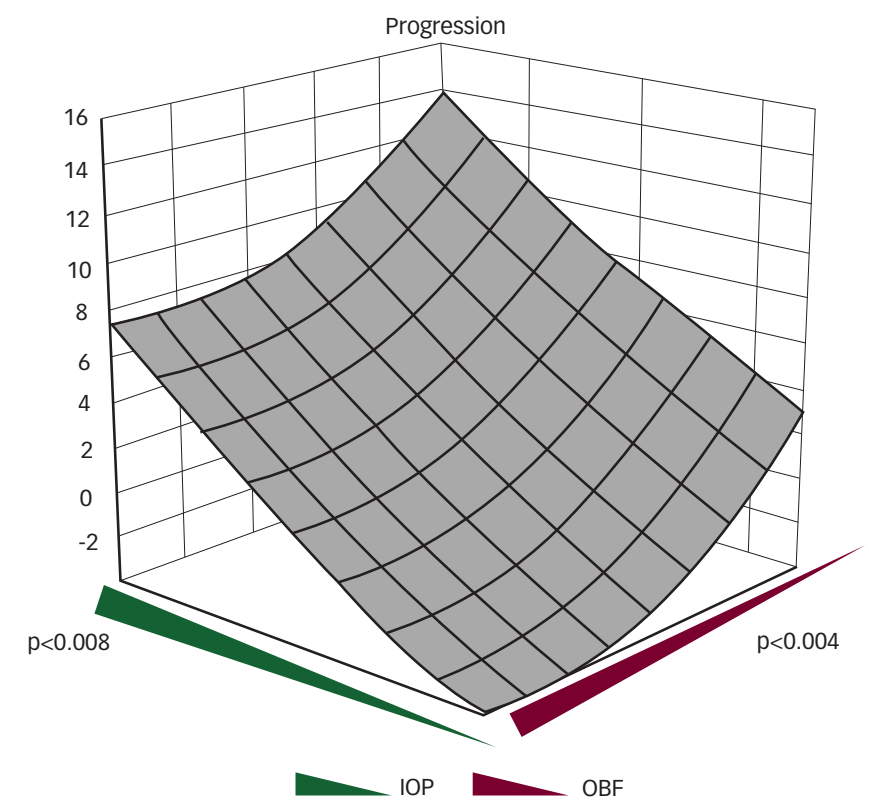

$I O P=$ intraocular pressure; $O B F=$ ocular blood flow Adapted with permission from Satilmis et al., 2003. ${ }^{5}$

with low body-mass index, and in subjects with high physical and mental activity. The leading symptoms are cold hands and feet and relatively low blood pressure, particularly at night., ${ }^{2,10}$ Interestingly, with a closer look, other signs, such as episodes of silent myocardial ischemia, altered beat-to-beat variation in electrocardiography, alteration in electroencephalography, altered gene expression in the lymphocytes, increased endothelin plasma levels, and others, can be found. When specifically asked for, these patients often indicate that they have a reduced feeling of thirst, altered drug sensitivity, increased smell sensitivity, and altered sleep behavior with a delayed sleep-onset time, in particular when they feel cold. All these symptoms tend to decline with age. ${ }^{14}$ 


\section{Figure 3: Schematic Illustration of the Blood-Brain Barrier}

A
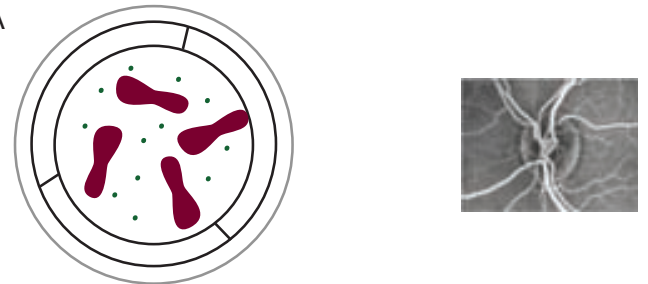

B

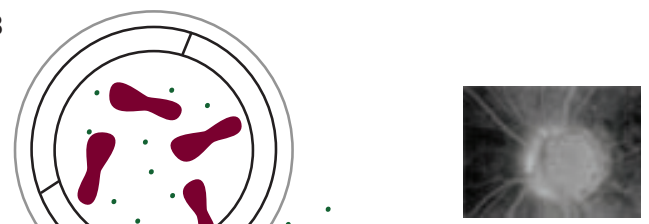

C

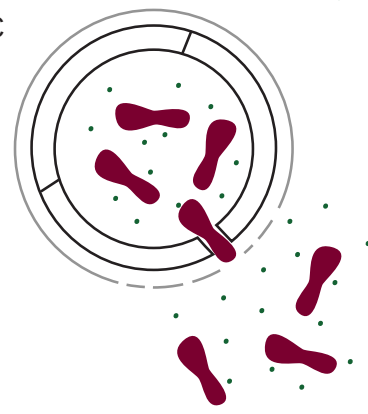

The microvessel (left) with its corresponding clinical picture (right). A: A physiological condition with minimal leakage of fluorescein; B: A glaucomatous optic nerve head with a disturbed blood-brain barrier, as a result of an increase in endothelin-1 and vascular endothelial growth factor (VEGF), which leads to dysfunction of tight junctions and an increase in fluorescein leakage; $C$ : If the basement membrane is simultaneously digested by metalloproteinase-9, erythrocytes may escape the vascular lumen, which leads to what is clinically observed as optic disk hemorrhages. Adapted with permission from Grieshaber et al., 2007. ${ }^{18}$

Figure 4: Retinal Veins and Retinal Arteries in the Lamina Cribrosa and at Arterial Venous Crossings
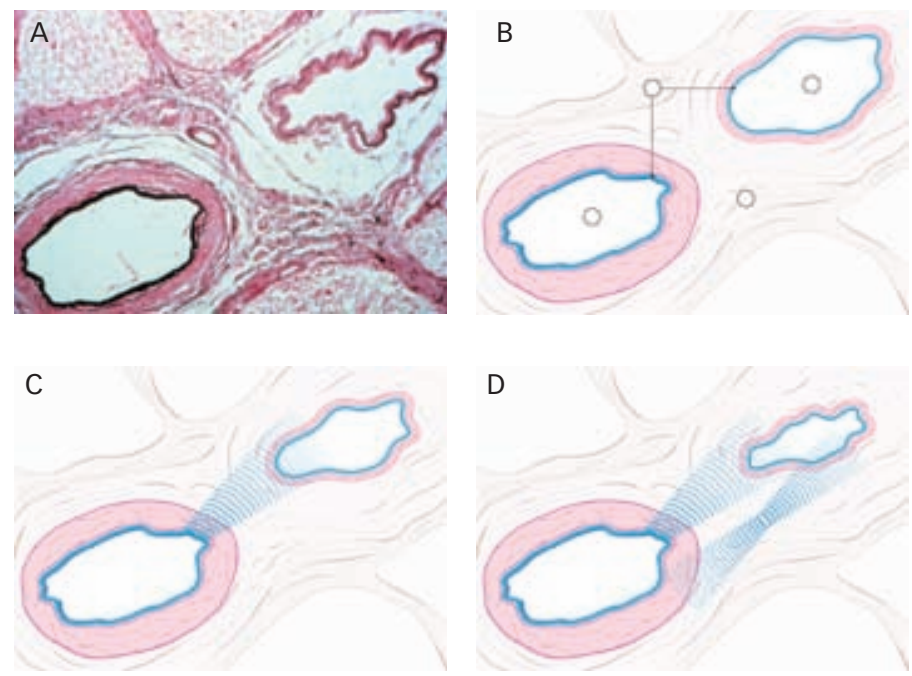

A: Retinal veins and retinal arteries are in close vicinity, in the lamina cibrosa and at arterial venous crossings; $B$ : A corresponding schema. Vasoactive substances reach the vein either from the arterial wall $(C)$ or from the (hypoxic) retinal tissue (D), which leads to constriction of the vein. 1 = artery; 2 = vein; 3 = adventitia; 4 = vascular endothelial cell laver. Reproduced with permission from Fraenkl et al., 2010.19
Figure 2: Activated Astrocytes Lead to Increased Backscatter of Light as Demonstrated in this Red-free Fundus Photo

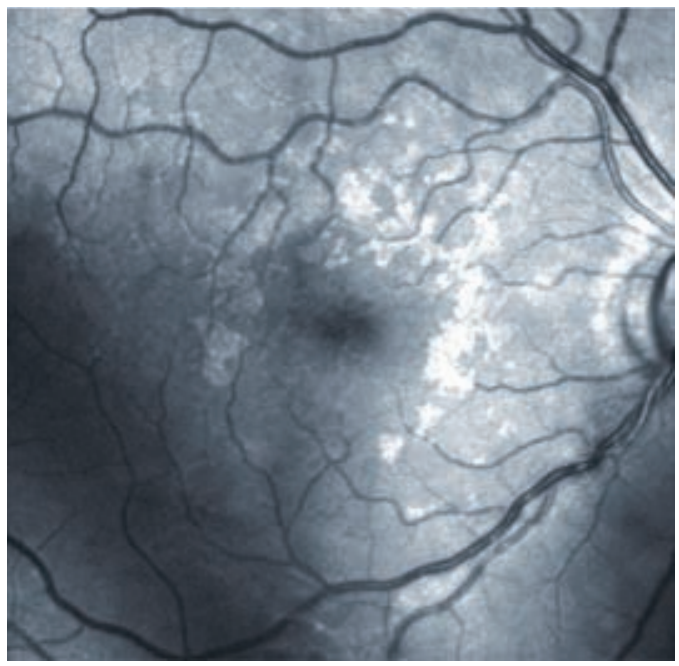

Reproduced with permission from Grieshaber et al., 2007."

Figure 5: Schematic Representation of the Concept of the Role of Unstable Ocular Perfusion in the Pathogenesis of Glaucomatous Damage

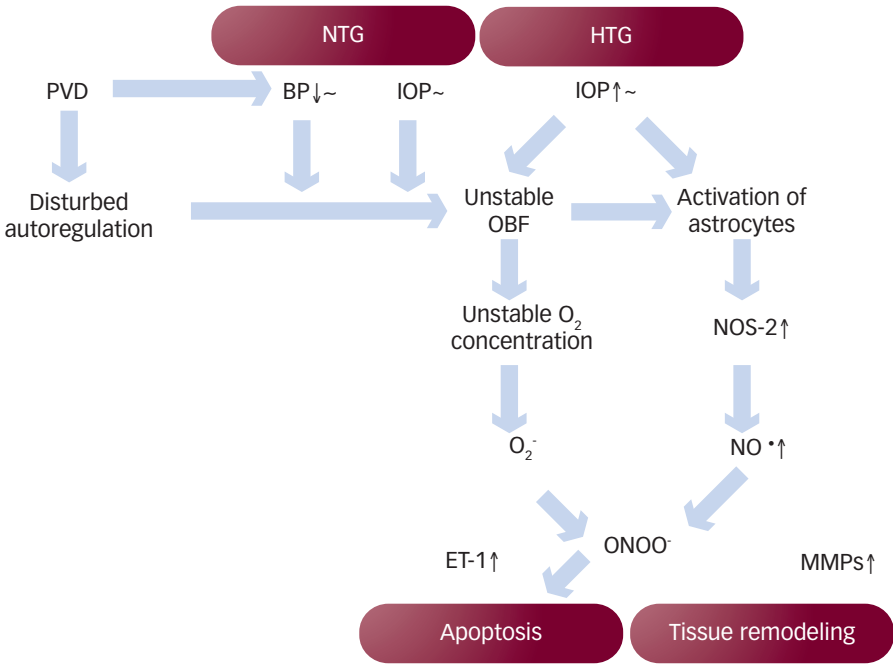

$B P=$ blood pressure; $E T-1=$ endothelin-1; HTG = high-tension glaucoma; IOP = intraocular pressure; $M M P=$ metalloproteinase; $N O=$ nitric oxide; NOS = nitric oxide synthase; $N T G=$ normal-tension glaucoma; $O B F=$ ocular blood flow; ONOO = peroxynitrite $P V D=$ primary vascular dysregulation

Adapted with permission from Survey of Ophthalmology. ${ }^{20}$

\section{How is Ocular Blood Flow in Primary Vascular Dysregulation Subjects?}

Pulse waves in retinal vessels propagate faster in subjects with PVD than in non-PVD subjects, which indicates that the rigidity of their retinal vessels is higher, despite their anatomically normal appearance. However, in these patients spatial irregularity of retinal vessels is increased, neurovascular coupling is decreased, and autoregulation of OBF is disturbed. ${ }^{15}$ The dysfunction of the autoregulation likely depicts the causal relationship between PVD and glaucomatous damage. 


\section{Why Does Unstable Ocular Blood Flow Lead to Glaucomatous Optic Neuropathy?}

Unstable OBF leads to unstable oxygen supply, which in turn triggers oxidative stress. An increased concentration of superoxide anions in an area where the production of nitric oxide (NO) is increased augments the production of the very damaging molecule peroxynitrite. Indeed, the production of NO is increased in astrocytes of these patients, activated either by mechanical stress or by endothelin. Unlike superoxide, NO diffuses easily into other cells and therefore also from astrocytes into neurons and their axons. ${ }^{16}$

\section{Can the Activation of the Astrocytes be Visualized Clinically?}

Activated astrocytes not only change their gene expression, but also their morphology. They lose their regular pattern and subsequently increase light scattering. This contributes to the blur that glaucoma patients often experience. The backscatter of light also leads to glinting spots in the retina observed by ophthalmologists (see Figure 2). ${ }^{17}$

\section{What is the Link between Ocular Blood Flow and Splinter Hemorrhages?}

Traditionally, splinter hemorrhages were considered to be a consequence of either microinfarction or small vessel rupture. However, we suggested that these hemorrhages are rather a consequence of a local breakdown of the blood-retinal or blood-brain barrier. ${ }^{18}$ If this barrier is malfunctioning, not only on the level of endothelial cells (because of increased concentrations of VEGF and endothelin), but simultaneously on the level of the basal membrane (because of increased concentration of metalloproteinase-9 [MMP-9]), then occasionally erythrocytes will escape from the vessels, which thus explains the clinical picture of hemorrhage (Figure 3).
The molecules mentioned above interfere with the barrier function and can be increased by diffusion from the circulating blood via fenestrated capillaries of the choroid into the optic nerve head and the adjacent retina or they can be produced - at least to some extent-locally in the retina. The latter is more relevant for retinal hemorrhages in diabetes than for optic nerve head hemorrhages in glaucoma patients. In other words, vascular dysregulation not only leads to an inappropriate vascular tone, but eventually also to a barrier dysfunction. It is therefore not surprising that the chance of damage progression is higher in areas where hemorrhages occur and that these hemorrhages occur more often in females and in NTG patients. Although hemorrhages occur more often in NTG patients, their frequency is reduced if oxygen supply is improved by reducing IOP.

\section{What is the Relationship between Retinal Vein Occlusion and Glaucoma?}

Retinal vein occlusions are found more often in glaucoma patients. Furthermore, both glaucomatous damage and vein occlusion occur more often in subjects with PVD. We suggested that the vein occlusion is not caused primarily by thrombus formation, but rather by a local dysregulation of the vein, either in the lamina cribrosa or at the arteriovenous crossing. ${ }^{19}$ This dysregulation again is likely a consequence of a local increase of vasoactive molecules, such as endothelin, either diffusing from the circulating blood or produced in the neural tissue of the retina (Figure 4). This, in turn, occurs particularly in hypoxic tissue. Therefore, a primary arterial dysfunction may lead to secondary venous dysfunction.

In summary, unstable oxygen supply caused by IOP or blood pressure fluctuations or disturbed autoregulation leads to increased oxidative stress, a main contributor to glaucomatous damage (Figure 5). ${ }^{20}$
1. Harris A, Kagemann L, Ehrlich R, et al., Measuring and interpreting ocular blood flow and metabolism in glaucoma, Can I Ophthalmol, 2008;43:328-36.

2. Mozaffarieh M, Flammer J, Ocular Blood Flow and Glaucomatous optic Neuropathy, Germany: Springer-Verlag Berlin Heidelberg, 2009.

3. Kaiser HJ, Schoetzau A, Stumpfig D, Flammer J, Blood-flow velocities of the extraocular vessels in patients with high-tension and normal-tension primary open-angle glaucoma, Am I Ophthalmol, 1997:123:320-7.

4. Gasser P, Flammer J, Blood-cell velocity in the nailfold capillaries of patients with normal-tension and high-tension glaucoma, Am I Ophthalmol, 1991;111:585-8.

5. Satilmis M, Orgul S, Doubler B, Flammer J, Rate of progression of glaucoma correlates with retrobulbar circulation and intraocular pressure, Am I Ophthalmol, 2003:135:664-9.

6. Tezel G, Wax MB, Hypoxia-inducible factor 1-alpha in the glaucomatous retina and optic nerve head, Arch Ophthalmol, 2004;122:1348-56

7. Haufschild T, Shaw SG, Kesselring J, Flammer J, Increased endothelin-1 plasma levels in patients with multiple sclerosis, J Neuroophthalmol, 2001:21:37-8.

8. Flammer J, Eppler E, Niesel P, Quantitative perimetry in the glaucoma patient without local visual field defects, Graefes Arch Clin Exp Ophthalmol, 1982;219:92-4.

9. Flammer J, Haefliger IO, Orgul S, Resink T, Vascular dysregulation: a principal risk factor for glaucomatous damage?, J Glaucoma, 1999;8:212-9.

10. Flammer J, Orgul S, Costa VP, et al., The impact of ocular blood flow in glaucoma, Prog Retin Eye Res, 2002;21:359-3.

11. Kaiser HJ, Flammer J, Graf T, Stumpfig D, Systemic blood pressure in glaucoma patients, Graefes Arch Clin Exp Ophthalmol, 1993;231:677-80

12. Sung KR, Lee $S$, Park SB, et al., Twenty-four hour ocular perfusion pressure fluctuation and risk of normal-tension glaucoma progression, Invest Ophthalmol Vis Sci, 2009:50:5266-74.

13. Stodtmeister $R$, The pulsation and the pressure of the central retinal vein and their relation to glaucoma damage and therapy, Klin Monbl Augenheilkd, 2008;225:632-6.

14. Flammer J, Pache M, Resink T, Vasospasm, its role in the pathogenesis of diseases with particular reference to the eye, Prog Retin Eye Res, 2001:20:319-49.

15. Gherghel D, Orgul S, Dubler B, et al., Is vascular regulation in the central retinal artery al-tered in persons with vasospasm?, Arch Ophthalmol, 1999;117:1359-62.

16. Liu B, Neufeld AH, Activation of epidermal growth factor receptor causes astrocytes to form cribriform structures, Glia, 2004:46:153-68.

17. Grieshaber MC, Orgul S, Schoetzau A, Flammer J, Relationship between retinal glial cell activation in glaucoma and vascular dysregulation, J Glaucoma, 2007;16:215-9.

18. Grieshaber MC, Flammer J, Does the blood-brain barrier play a role in glaucoma?, Surv Ophthalmol, 2007;52(Suppl. 2):S115-21.

19. Fraenkl SA, Mozaffarieh M, Flammer J, Retinal vein occlusions: the potential impact of a dysregulation of the retinal veins, EPMA J, 2010;1:253-61.

20. Flammer J, Mozaffarieh $\mathrm{M}$, What is the present pathogenetic concept of glaucomatous optic neuropathy?, Surv Ophthalmol, 2007;52(Suppl. 2):S162-73. 